\title{
FREDERIKS MEDAL
}

The 14-th European Conference on Liquid Crystals was held in Moscow, Russia, on June 25-30, 2017. The conference was dedicated to the 90-th anniversary of Frederiks effect which has provided the basis for the display technology. Vsevolod Frederiks (Freedericksz) was a Russian physicist, the founder of the liquid crystal scientific school in Russia. Such dedication gives us a great opportunity to speak about the Frederiks medal, which was established by Russian Liquid Crystal Society «Sodruzhestvo» («Commonwealth») in 1996 as the highest scientific award in the field of liquid crystals. The statute of Frederiks medal was elaborated by Professor Anatoly Sonin.

The main items of this statute are the following:

1. The Frederiks medal is awarded for the outstanding scientific results in the field of liquid crystals;

2. Every year two Frederiks medals are awarded: one in the field of liquid crystal chemistry, another one in the field of liquid crystal physics;
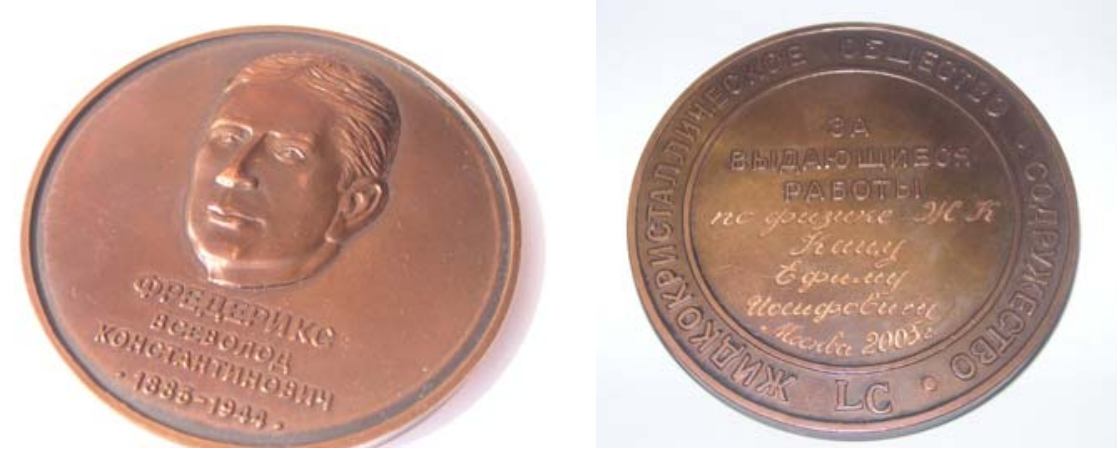
one - to the foreign scientists; reversed situation should be; years ago; members; person; (Fig. 1, b).
3. One of the medals is to the Russian scientists (or to the scientists of former Soviet Union) another

4. If one year a Russian (or former Soviet) physicist and a foreign chemist were awarded, next year the

5. The pretender has to be actively working person, not the one who had outstanding results many

6. Each member of the Russian Liquid Crystal Society can apply two candidates for the medal every year; the decision is taken by a simple majority vote of the «Sodruzhestvo» board

7. The medal is done from cooper alloy and has two sides: on one side - the profile of Frederiks, on another side - the text and the name of awarded

8. Along with the medal (Fig. 1,a), the awarded person receives a diploma created by painter

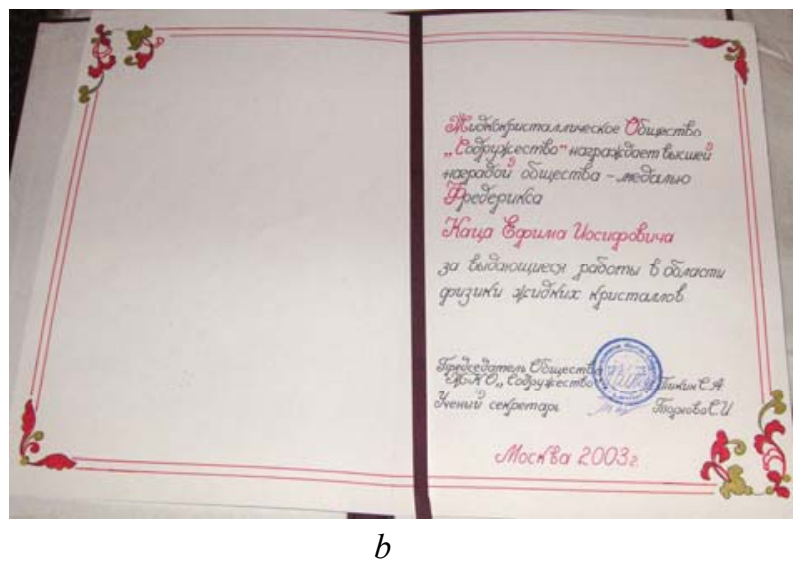

Liquid Crystal Society «Sodruzhestvo» (Commonwealth) awarded with the highest prize of our Society - Frederiks medal Kats Efim Iosifovich

for the outstanding results in the field of Liquid Crystal Physics.

President of Liquid Crystal

Society "Sodruzhestvo»

Pikin S.A.

Scientific Secretary

Moscow 2003

Fig. 1. The obverse of the medal consists of a representation of a profile of Vsevolod Frederiks. The reverse side has the text and the recipient's name (a); the diploma of Prof. E. Kats $(b)$ 
The statute of the medal was approved by Russian Liquid Crystal Society members on the 10-th meeting of Society - 24 October 1996.

The medal was minted in St-Petersburg on the Russian Federation Mint. The sponsors were: Professor Victor Titov and Professor Alexander Ivashchenko.

The first awarded persons were: member correspondent of Russian Academy of Sciences,

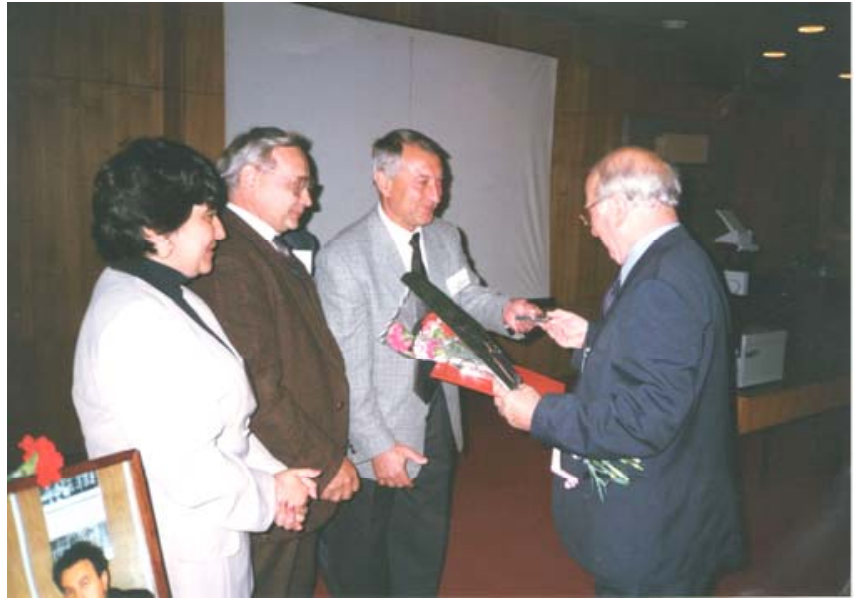

Fig. 2. Medal awards ceremony for Prof. George Gray (UK). From left to right - the members of «Sodruzhestvo» board Dr. S. Torgova, Prof. S. Pikin, Prof. E. Ryumtsev (1997)
Professor Victor Tsvetkov (in the field of liquid crystal physics) and Professor Georg Gray (in the field of liquid crystal chemistry, England). The awards were handed out on the 11-th meeting of Society dedicated to the memory of Professor Victor Titov, 21-st of May 1997 in Old Peterhof (close to St.-Petersburg) (Fig. 2).

So far 40 scientists from different countries were awarded the medal (Fig. 3, 4, Table).

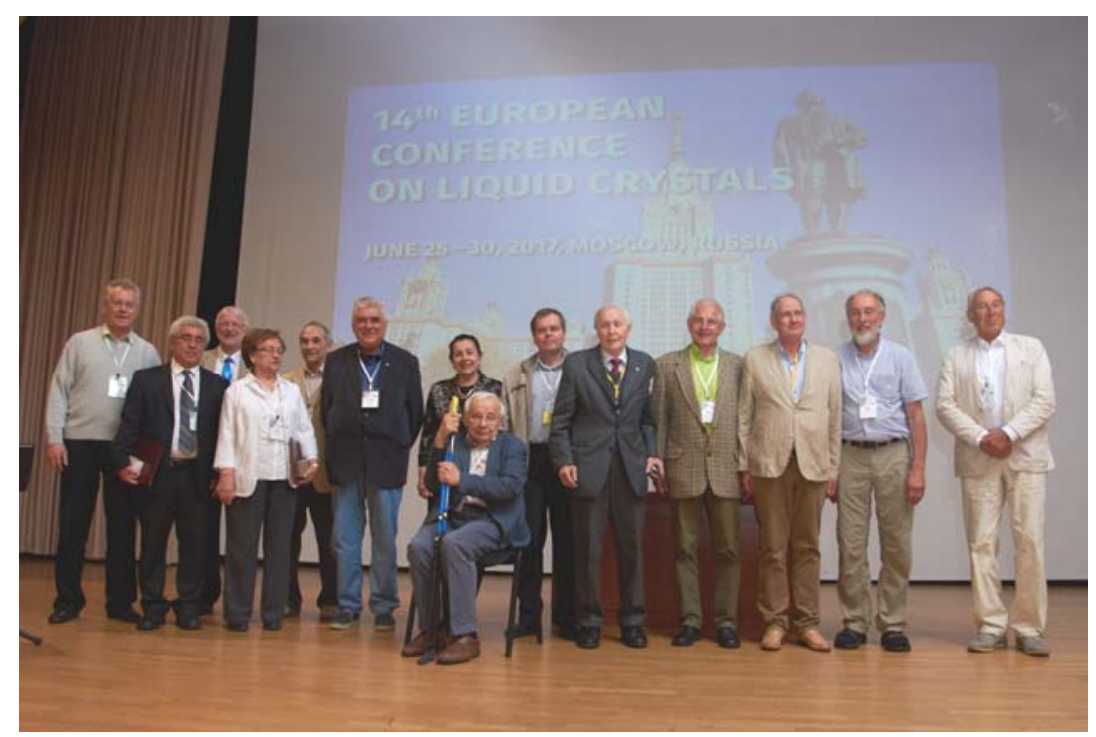

Fig. 4. Previously awarded scientists - the paricipants of the 14-th ECLC (2017):

From left to right - Prof. E. Averyanov (Russia), Prof. N. Tabiryan (USA), Prof. John Goodby (UK), Prof. G. Zharkova (Russia), Prof. L. Blinov (Russia), Prof. A. Petrov (Bulgaria), Prof. N. Usol'tseva (Russia), Prof. S. Pikin (Russia), Prof. S. Palto (Russia), Prof. V. Shibaev (Russia), Prof. V. Bezborodov (Belarus), Prof. B. Ostrovskii (Russia), Prof. N. Clark (USA), Prof. S. Lagerwall (Sweden) 
The list of awarded scientists

\begin{tabular}{|c|c|c|c|}
\hline № & Year & Surname, first name & Section of science \\
\hline 1. & 1997 & $\begin{array}{l}\text { Tsvetkov Victor (Russia) } \\
\text { Gray George (UK) }\end{array}$ & $\begin{array}{c}\text { Physics } \\
\text { Chemistry }\end{array}$ \\
\hline 2. & 1998 & $\begin{array}{l}\text { Zaupe Alfred (Germany) } \\
\text { Shibaev Valery (Russia) }\end{array}$ & $\begin{array}{l}\text { Physics } \\
\text { Chemistry }\end{array}$ \\
\hline 3. & 1999 & $\begin{array}{l}\text { Blinov Lev (Russia) } \\
\text { Demus Dietrich (Germany) }\end{array}$ & $\begin{array}{l}\text { Physics } \\
\text { Chemistry }\end{array}$ \\
\hline 4. & 2000 & $\begin{array}{l}\text { Chandrasekhar Subrahmanyan (India) } \\
\text { Sonin Anatoly (Russia) }\end{array}$ & $\begin{array}{l}\text { Physics } \\
\text { Chemistry }\end{array}$ \\
\hline 5. & 2001 & $\begin{array}{l}\text { Pikin Sergey (Russia) } \\
\text { Neubert Mary (USA) }\end{array}$ & $\begin{array}{l}\text { Physics } \\
\text { Chemistry }\end{array}$ \\
\hline 6. & 2002 & $\begin{array}{l}\text { Lagerwall Sven (Sweden) } \\
\text { Kutulya Lidia (Ukraine) }\end{array}$ & $\begin{array}{l}\text { Physics } \\
\text { Chemistry }\end{array}$ \\
\hline 7. & 2003 & $\begin{array}{l}\text { Kats Efim (Russia) } \\
\text { Dabrowski Roman (Poland) }\end{array}$ & $\begin{array}{l}\text { Physics } \\
\text { Chemistry }\end{array}$ \\
\hline 8. & 2004 & $\begin{array}{l}\text { Petrov Alexander (Bulgaria) } \\
\text { Usol'tseva Nadezhda (Russia) }\end{array}$ & $\begin{array}{l}\text { Physics } \\
\text { Chemistry }\end{array}$ \\
\hline 9. & 2005 & $\begin{array}{l}\text { Chilaya Guram (Georgia) } \\
\text { Eidenschink Rudolf (Germany) }\end{array}$ & $\begin{array}{l}\text { Physics } \\
\text { Chemistry }\end{array}$ \\
\hline 10. & 2006 & $\begin{array}{l}\text { Luckhurst Geoffrey (UK) } \\
\text { Bezborodov Vladimir (Belarus) }\end{array}$ & $\begin{array}{l}\text { Physics } \\
\text { Chemistry }\end{array}$ \\
\hline 11. & 2007 & $\begin{array}{l}\text { Ryumtsev Evgenii (Russia) } \\
\text { Zaschke Horst (Germany) }\end{array}$ & $\begin{array}{l}\text { Physics } \\
\text { Chemistry }\end{array}$ \\
\hline 12. & 2008 & $\begin{array}{l}\text { Clark Noel (USA) } \\
\text { Evdokimov Yuri (Russia) }\end{array}$ & $\begin{array}{l}\text { Physics } \\
\text { Chemistry }\end{array}$ \\
\hline 13. & 2009 & $\begin{array}{l}\text { Reznikov Yuri (Ukraine) } \\
\text { Finkelman Haino (Germany) }\end{array}$ & $\begin{array}{l}\text { Physics } \\
\text { Chemistry }\end{array}$ \\
\hline 14. & 2010 & $\begin{array}{l}\text { Schadt Martin (Switzerland) } \\
\text { Karamysheva Ludmila (Russia) }\end{array}$ & $\begin{array}{l}\text { Physics } \\
\text { Chemistry }\end{array}$ \\
\hline 15. & 2011 & $\begin{array}{l}\text { Palto Sergey (Russia) } \\
\text { Tschierske Carsten (Germany) }\end{array}$ & $\begin{array}{l}\text { Physics } \\
\text { Chemistry }\end{array}$ \\
\hline 16. & 2012 & $\begin{array}{l}\text { De Jeu Wim H. (Germany) } \\
\text { Bolotin Boris (Russia) }\end{array}$ & $\begin{array}{l}\text { Physics } \\
\text { Chemistry }\end{array}$ \\
\hline 17. & 2013 & $\begin{array}{l}\text { Ostrovskii Boris (Russia) } \\
\text { Haase Wolfgang (Germany) }\end{array}$ & $\begin{array}{l}\text { Physics } \\
\text { Chemistry }\end{array}$ \\
\hline 18. & 2014 & $\begin{array}{l}\text { Takezoe Hideo (Japan) } \\
\text { Galyametdinov Yuri (Russia) }\end{array}$ & $\begin{array}{l}\text { Physics } \\
\text { Chemistry }\end{array}$ \\
\hline 19. & 2015 & $\begin{array}{l}\text { Averyanov Evgenii (Russia) } \\
\text { Goodby John (UK) }\end{array}$ & $\begin{array}{l}\text { Physics } \\
\text { Chemistry }\end{array}$ \\
\hline 20. & 2016 & $\begin{array}{l}\text { Tabiryan Nelson (USA) } \\
\text { Zharkova Galina (Russia) }\end{array}$ & $\begin{array}{l}\text { Physics } \\
\text { Chemistry }\end{array}$ \\
\hline
\end{tabular}

DOI: 10.18083/LCApp1.2017.3.93 